Tropical Journal of Pharmaceutical Research March 2020; 19 (3): 481-487

ISSN: $1596-5996$ (print); 1596-9827 (electronic)

(C) Pharmacotherapy Group, Faculty of Pharmacy, University of Benin, Benin City, 300001 Nigeria.

Available online at http://www.tjpr.org

Original Research Article

http://dx.doi.org/10.4314/tjpr.v19i3.4

\title{
Lysine demethylase 1A exacerbates LPS-induced inflammation of vascular smooth muscle cells through modulation of NF-KB activation
}

\author{
Ling Liang ${ }^{1,2}$, Mingliang Sun ${ }^{3}$, Zhongquan $\mathrm{Qi}^{4 *}$, Weihua $\mathrm{Li}^{1,2}$ \\ ${ }^{1}$ Department of Cardiology, The First Affiliated Hospital of Xiamen University. Xiamen, Fujian Province 361000, ${ }^{2}$ Department of \\ Cardiology, First Clinical Medical College of Fujian Medical University. Xiamen, Fujian Province 350000, ${ }^{3}$ Department of Plastic \\ Cosmetic Surgery, Women and Children's Hospital Affiliated with Xiamen University. Xiamen, Fujian Province 361003, ${ }^{4}$ Institute \\ of Organ Transplantation, Xiamen University, Xiamen, Fujian Province 361005, China
}

*For correspondence: Email: ZhongquanQiwry@163.com; Tel: +86-592-2181680

Sent for review: 7 November 2019

Revised accepted: 28 February 2020

\begin{abstract}
Purpose: To study the effect of lysine demethylase 1A (LSD1) on inflammatory responses of vascular smooth muscle cells (VSMCs), and investigate the mechanism.

Methods: VSMCs were treated with lipopolysaccharide (LPS). Overexpression and knockdown of LSD1 in VSMCs were performed by transfecting with LSD1 overexpression plasmid and small interfering RNAs (siRNAs), respectively. Western blot and quantitative real-time polymerase chain reaction (qRT$P C R$ ) were used to measure protein and mRNA levels. Enzyme-linked immunosorbent (ELISA) assay was used to determine the levels of inflammatory cytokines.

Results: Phosphorylation of LSD1 ( $p$-LSD1) was significantly increased in LPS-induced VSMCs. Monocyte chemoattractant protein-1 and IL-6 levels were also increased by LPS, but attenuated by LSD1 knockdown in VSMCs. Activation of NF-KB was increased by LPS, but was also decreased by LSD1 knockdown. Level of methylated p65 (p65-me) in VSMCs was increased by treatment with SET7/9 (p65 methyltransferase), but this effect was attenuated by overexpression of LSD1. Besides, the increased levels of MCP-1 and IL-6 induced by overexpression of LSD1 were reversed by NF-KB signaling inhibitor, PDTC.

Conclusion: LSD1 exacerbates LPS-induced inflammation of VSMCs through NF-KB activation via p65 demethylation, which indicates that LSD1 might be a potential target for the treatment of cardiovascular diseases.
\end{abstract}

Keywords: Vascular smooth muscle cells, Lysine demethylase 1A, Phosphorylation, NF-kB, p65, Demethylation

\begin{abstract}
This is an Open Access article that uses a fund-ing model which does not charge readers or their institutions for access and distributed under the terms of the Creative Commons Attribution License (http://creativecommons.org/licenses/by/4.0) and the Budapest Open Access Initiative (http://www.budapestopenaccessinitiative.org/read), which permit unrestricted use, distribution, and reproduction in any medium, provided the original work is properly credited.

Tropical Journal of Pharmaceutical Research is indexed by Science Citation Index (SciSearch), Scopus, International Pharmaceutical Abstract, Chemical Abstracts, Embase, Index Copernicus, EBSCO, African Index Medicus, JournalSeek, Journal Citation Reports/Science Edition, Directory of Open Access Journals (DOAJ), African Journal Online, Bioline International, Open-J-Gate and Pharmacy Abstracts
\end{abstract}

\section{INTRODUCTION}

Vascular smooth muscle cells (VSMCs) are main components of the vascular wall, contributing primarily to maintain the stability of blood flow and pressure [1,2]. Abnormal proliferation and inflammatory response of VSMCs may result in various vascular diseases such as vascular

(c) 2020 The authors. This work is licensed under the Creative Commons Attribution 4.0 International License 
stenosis and atherosclerosis after vessel transplant $[3,4]$. During inflammation induced by lipopolysaccharide (LPS) or other factors, VSMCs produce extensive inflammatory cytokines related to vascular lesions, acceleration of atherosclerosis, and formation of susceptible plaques $[5,6]$. Therefore, it is of great importance to investigate the mechanism underlying excessive inflammation in VSMCs for the diagnosis and treatment of cardiovascular diseases.

Lysine demethylase 1A (LSD1), also known as lysine (K)-specific demethylase 1A (KDM1A), could demethylate histone $\mathrm{H} 3$ [7] and is involved in the regulation of inflammation. In hepatitis $B$ virus-associated glomerulonephritis, LSD1 level was notably increased, and promoted the release of proinflammatory mediators and Toll-Like Receptor 4 [8]. Therefore, LSD1 is considered a regulator of renal inflammation [8].

In a previous study, protein kinase Ca (PKCa)LSD1-nuclear factor-kB (NF-kB) signaling was a critical for LPS-induced inflammatory response in mice and bone marrow-derived macrophages (Raw264.7 cells) [9]. During inflammatory response, increased LSD1 phosphorylation is induced by $\mathrm{PKCa}$, and LSD1 phosphorylation accelerated the demethylation of p65 and increased the stability of p65 [9]. The inflammatory injury was alleviated after inhibition of LSD1 or PKCa activity [9]. Therefore, LSD1 might be a novel target for regulating the inflammatory response by NF-KB signaling. However, it is unclear whether LSD1 affects the inflammatory response of VSMCs.

Therefore, this study is intended to investigate the effect of LSD1 on excessive inflammatory responses of VSMCs and reveal the underlying regulatory mechanism of this process.

\section{EXPERIMENTAL}

\section{Cell culture}

VSMCs were maintained in a $\mathrm{CO}_{2}$ incubator (5 $\%$; $37{ }^{\circ} \mathrm{C}$ ) using Dulbecco's Modified Eagle's

Table 1: Sequence of the primers for PCR
Medium (DMEM) with fetal bovine serum (10\%, FBS), penicillin $(100 \mathrm{U} / \mathrm{ml})$, and streptomycin (100 $\mu \mathrm{g} / \mathrm{ml}$; Gibco, Grand Island, NY, USA). To establish an inflammatory model of VSMCs, VSMCs were treated with LPS $(10,100$, or 1000 $\mathrm{ng} / \mathrm{ml}$ ) for, 30,60 or $120 \mathrm{~min}$. In indicated experiments, VSMCs were also treated with 80 $\mu \mathrm{mol} / \mathrm{l}$ ammonium pyrrolidinedithiocarbamate (PDTC; NF-kB inhibitor).

\section{RNA extraction and quantitative real-time polymerase chain reaction (QRT-PCR)}

Total RNA was extracted from VSMCs using RNeasy Mini Kit (Qiagen, Valencia, CA) and measured by s NanoDrop spectrophotometer (ThermoFisher Scientific, Waltham, MA). Complementary DNA (cDNA) was performed by reverse transcription and $\mathrm{qPCR}$ was performed with the following sequences of primers (Table 1). GAPDH was used as control.

\section{Western blot}

Total protein was extracted from VSMCs using radioimmunoprecipitation assay and the concentration of the extracted protein was quantified. Forty micrograms of extracted protein was subjected to $12 \%$ SDS-PAGE and transferred to a PVDF membrane, which was then blocked by $4 \%$ non-fat milk for $1 \mathrm{~h}$. The PVDF membranes were incubated with primary antibodies for $12 \mathrm{~h}$ and secondary antibody (antirabbit IgG antibody) for $1 \mathrm{~h}$. The primary antibodies were anti-LSD1 antibody (1:500, Abcam), anti-p-LSD1 antibody (1:500, Abcam), anti-p65 antibody (1:500, Abcam), and anti-lamin A/C antibody (1:500, Abcam). The membranes were visualized using ECL Plus reagents, and the bands were quantified.

\section{Enzyme-linked immunosorbent assay (ELISA)}

The cell supernatants of VSMCs were collected and MCP-1 and interleukin (IL)-6 levels were detected using ELISA kits (Abcam, Cambridge, MA).

\begin{tabular}{lll}
\hline Gene name & Forward & Reverse \\
\hline LSD1 & 5'-GAGGGAATCCTATGGCTGTGG-3' & 5'-AAACTCTTGCTCTACCATTTCATCT- \\
& & 3' \\
MCP-1 & 5'-GCACGTTTCAGTGAGCATCG-3' & 5'-CAGCAGAGTGGGTGGATTCT-3' \\
IL-6 & 5'-TACCCCCAGGAGAAGATTCCA-3' & 5'-CCTCTTTGCTGCTTTCACACA-3' \\
GAPDH & 5'-TGAGAACGGGAAGCTTGTCA-3' & 5'-GCATCGCCCCACTTGATTTT-3'
\end{tabular}




\section{Cell transfection}

Small interfering RNA (siRNA) (siNC), siRNA for LSD1 (siLSD1), and LSD1 overexpression plasmid were synthesized at Shanghai GenePharma Co., Ltd. For cell transfection, $4 \times$ $10^{5}$ VSMCs were seeded into each well of a 6well plate and cultured in DMEM for $24 \mathrm{~h}$. The VSMCs were then transfected with siNC, siLSD1, or LSD1 overexpression plasmid using Lipofectamine 3000 reagent (ThermoFisher Scientific, Waltham, MA). VSMCs were collected after $48 \mathrm{~h}$ of transfection.

\section{Statistical analysis}

Statistical analysis was performed using SPSS 22.0 (Chicago, IL, USA) and GraphPad Prism 7 (La Jolla, CA, USA). All data were shown as mean \pm standard deviation (SD). Comparison between two groups or among multiple groups was made using Student's t-test or one-way analysis of variance (ANOVA). A value of $p<$ 0.05 was considered statistically significant. Each experiment was repeated at least three times.

\section{RESULTS}

\section{LPS-induced LSD1 phosphorylation in VSMCS}

To investigate the role of LSD1 in the LPSinduced inflammatory response of VSMCs, the phosphorylation level of LSD1 (p- LSD1) in VSMCs that treated with LPS was determined. As shown in Figure1A, western blot results showed no significant differences in total LSD1 levels between VSMCs treated with LPS and that without LPS treatment. The protein level ofpLSD1 was significantly higher in VSMCs treated with 100 or $1000 \mathrm{ng} / \mathrm{mL}$ LPS, while p-LSD1 protein expression was not affected by $10 \mathrm{ng} / \mathrm{mL}$ LPS as compared to that without LPS treatment. Therefore, the ratio of p-LSD1/LSD1 was significantly higher in the 100 and $1000 \mathrm{ng} / \mathrm{mL}$ LPS-treated groups than in the control group $(p<$ 0.01 ).

The protein expression of $p$-LSD1 was examined in VSMCs that treated with $1000 \mathrm{ng} / \mathrm{mL}$ LPS for 30,60 , and $120 \mathrm{~min}$. As shown in Figure 1B, western blot results showed no significant differences in total LSD1 levels among various groups. However, levels of p-LSD1 were significantly increased in VSMCs treated with $1000 \mathrm{ng} / \mathrm{mL}$ LPS for 60 and $120 \mathrm{~min}$ when compared to that without LPS treatment. Hence, the ratio of $p$-LSD1/LSD1 was significantly higher in groups that treated with 60 and 120 min than that in the control group $(p<0.01)$.
These results indicated that high concentrations of LPS (100 and $1000 \mathrm{ng} / \mathrm{mL}$ ) and long treatment time significantly increased p-LSD1 protein expression.

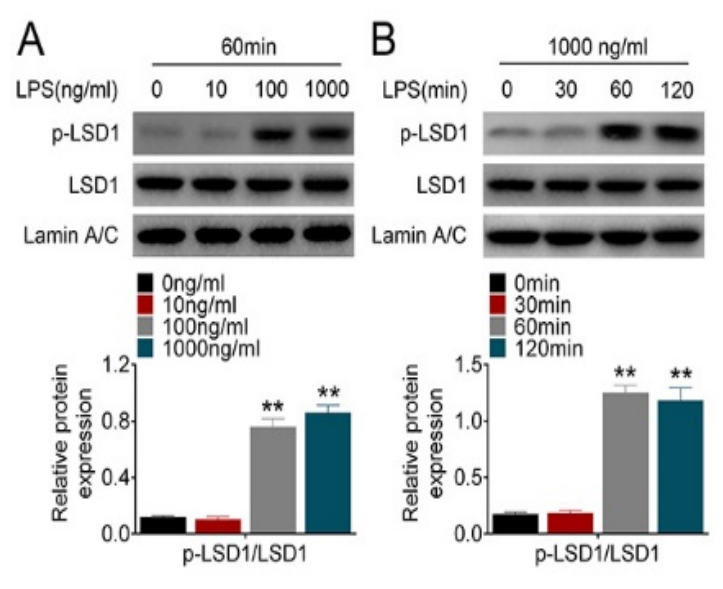

Figure 1: Effect of LPS on LSD1 phosphorylation in VSMCs. VSMCs were treated with 10,100 , or 1000 $\mathrm{ng} / \mathrm{ml} \mathrm{LPS} \mathrm{for} 60 \mathrm{~min}(\mathrm{~A})$ and $1000 \mathrm{ng} / \mathrm{ml}$ LPS for 30 , 60 , or $120 \mathrm{~min}(\mathrm{~B})$, and were assessed by western blotting; ${ }^{* *} p<0.01$ compared to control group

\section{Knockdown of LSD1 attenuated LPS- induced inflammation and inhibited NF- KB activation in VSMCS}

The levels of inflammatory factors MCP-1, IL-6 and p65 were assessed in LPS-induced VSMCs after knockdown of LSD1.Results from qRT-PCR and western blot showed that the mRNA and protein levels of LSD1were significantly reduced in VSMCs that transfected with siLSD1-1 or siLSD1-2 when compared to that transfected with siNC $(p<0.05$, Figure $2 A$ and B). The efficiency of siLSD1-2 was higher than that of siLSD1-1, which was thus used in subsequent experiments.

Next, the levels of inflammatory factors MCP-1 and IL-6 were determined in LPS-induced VSMCs transfected with siLSD1. Results from qRT-PCR and ELISA assay showed that levels of MCP-1 and IL-6 mRNA and protein were significantly higher in LPS-induced VSMCs when compared to the non-LPS-treated group ( $p<$ 0.01 ) and that MCP-1 and IL-6 levels were decreased after VSMCs were transfected with siLSD1 $(p<0.01$, Figure $2 \mathrm{C}$ and D).

Results also showed that the level of p65 was significantly increased in VSMCs induced by LPS compared to the control group, while its level was significantly decreased when transfected with siLSD1 (all $p<0.01$, Figure $2 \mathrm{E}$ ). In addition, the level of $p$-LSD1 in LPS-induced VSMCs was increased compared to the control group, while its level was decreased in LPS-induced VSMCs 
after transfected with siLSD1. Therefore, the ratio of $p$-LSD1/LSD1 was significantly higher in LPSinduced VSMCs, which was significantly after transfected with siLSD1 $(p<0.01$. These results indicated that knockdown of LSD1 attenuated LPS-induced inflammation in VSMCs and inhibited NF-KB activation.

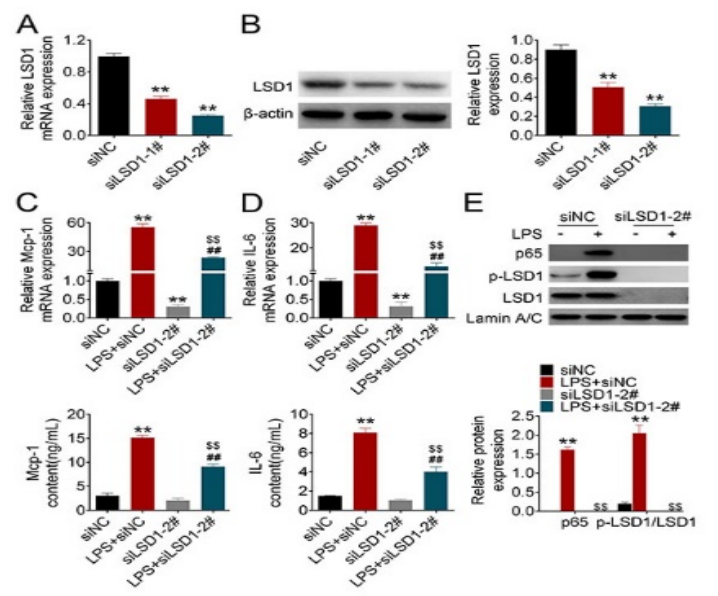

Figure 2: Effect of LSD1 knockdown on the expression of MCP-1, IL-6 and p65in LPS-treated VSMCs. VSMCs were transfected with two kinds of siLSD1, and the mRNA (A) and protein (B) levels of LSD1 were determined using qRT-PCR and Western blot. The levels of MCP-1 (C) and IL-6 (D) were determined using qRT-PCR and ELISA, and the levels of p65, LSD1, and p-LSD1 were quantified using Western blot $(\mathrm{E}) .{ }^{* *} p<0.01$ compared to siNC group; ${ }^{\#} p<0.01$ compared to the siLSD1 group; ${ }^{\$ \$} p<0.01$ compared to LPS + siNC group

\section{Knockdown of LSD1 inhibited NF-KB activation via p65 demethylation}

To p65 levels were examined in VSMCs that transfected with siLSD1 and treated with LPS for different time periods. The protein level of p65 in VSMCs treated with LPS for 30,60 , and 120 min were significantly increased when compared to that without LPS treatment ( $p<0.01$, Figure $3 \mathrm{~A})$. However, the p65 levels in VSMCs that treated with in LPS $(30,60$, and $120 \mathrm{~min})$ and transfected with si-LSD1 were significantly decreased when compared to that transfected with si-NC and treated with LPS $(p<0.01$, Figure 3 A). The levels ofp-LSD1 were also increased in LPS-treated groups 30, 60, and $120 \mathrm{~min}$ ), while this upregulation was reversed by knockdown of LSD1 $(p<0.01)$. Therefore, the ratio of $p$ LSD1/LSD1 was significantly increased in LPStreated VSMCs $(30,60$, and $120 \mathrm{~min})$, but this effect was significantly decreased by knockdown of LSD1 ( $p<0.01$, Figure 3 A).

For further analysis, VSMCs were transfected with LSD1 overexpression plasmid. Results from
qRT-PCR showed that the expression of LSD1 was significantly increased in VSMCs transfected with LSD1 overexpression plasmid when compared to control group ( $p<0.01$, Figure $3 \mathrm{~B}$ ). The VSMCs were then treated with LPS (1000 $\mathrm{ng} / \mathrm{mL}$ ) and SET7/9, a p65 methyltransferase, to determine whether p65 methylation was involved in the regulatory effects of LSD1 on NF-KB activation. Western blot results showed that methylated p65 (p65-me) levels were clearly increased in VSMCs treated with SET7/9 as compared to control group and that this phenomenon was abolished by overexpression of LSD1 (Figure $3 \mathrm{C}$ ).

Conversely, p65 level was clearly decreased in VSMCs treated with SET7/9 when compared to the control group, whereas p65 levels were increased in VSMCs that co-treated with SET7/9 and LSD1 plasmid compared to that treated with SET7/9 alone. The ratio of p65-me/p65 was significantly higher in VSMCs treated with SET7/9, and this effect was abolished by LSD1 overexpression $(p<0.01)$. In addition, the protein levels of $p$-LSD1 and LSD1 were increased in LPS-induced VSMCs co-treated with SET7/9 and LSD1 plasmid compared to that treated with SET7/9 alone. The ratio of $p$-LSD1/LSD1 showed no significant differences among four groups. These results indicated that knockdown of LSD1 inhibited NF-kB activation through p65 demethylation.

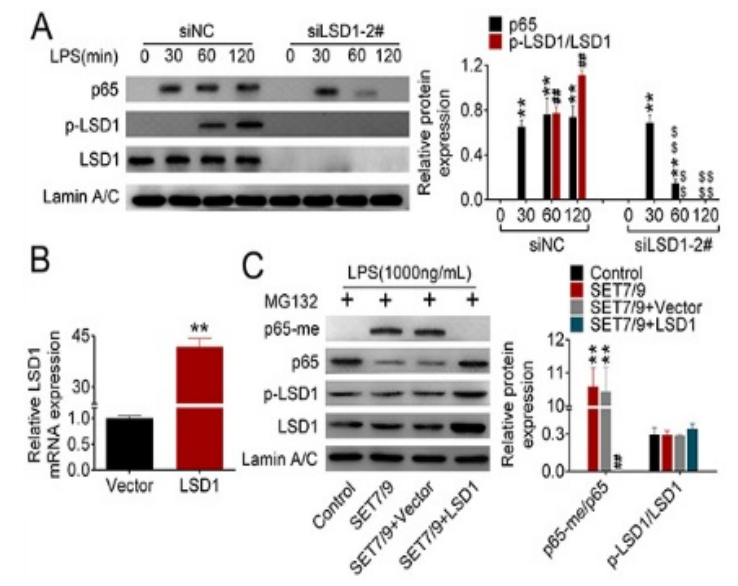

Figure 3: Knockdown of LSD1 inhibited NF-KB activation through p65 demethylation. (A) VSMCs were treated with $1000 \mathrm{ng} / \mathrm{ml}$ LPS for $0,30,60$, or 120 min and transfected with siLSD1, and the protein levels of p65, p-LSD1, and LSD1 were determined using Western blot. (B) VSMCs were transfected with LSD1 overexpression plasmid, and the mRNA levels of LSD1 were measured using qRT-PCR. (C) VSMCs were transfected with LSD1 overexpression plasmid and treated with SET7/9, and levels of p65-me, p65, LSD1, and p-LSD1were determined using Western

Trop J Pharm Res, March 2020; 19(3): 484 
blot; ${ }^{* *} p<0.01$ compared to control group; ${ }^{\# \#} p<0.01$ compared to SRT7/9 + vector group

\section{LSD1 exacerbated VSMC inflammation by activation of NF-kB}

To study whether LSD1 could regulate inflammation in VSMCs by NF-KB activation, LPS-treated VSMCs were treated with PDTC, an inhibitor of NF-kB. Results showed that p65 levels were significantly increased by LSD1 overexpression, which was reversed by PDTC ( $p$ $<0.05$, Figure 4 A). In addition, qRT-PCR and ELISA results showed that the levels of MCP-1 and IL-6 were also significantly increased by LSD1 overexpression when compared to the control group ( $p<0.01$, Figure $4 \mathrm{~B}$ and $\mathrm{C})$. After treated with PDTC, the mRNA and protein levels of MCP-1 $(p<0.05)$ and IL-6 $(p<0.01)$ significantly decreased (Figure $4 \mathrm{~B}$ and $\mathrm{C}$ ). These results indicated that LSD1 exacerbated inflammation in VSMCs by activation of NF-kB.

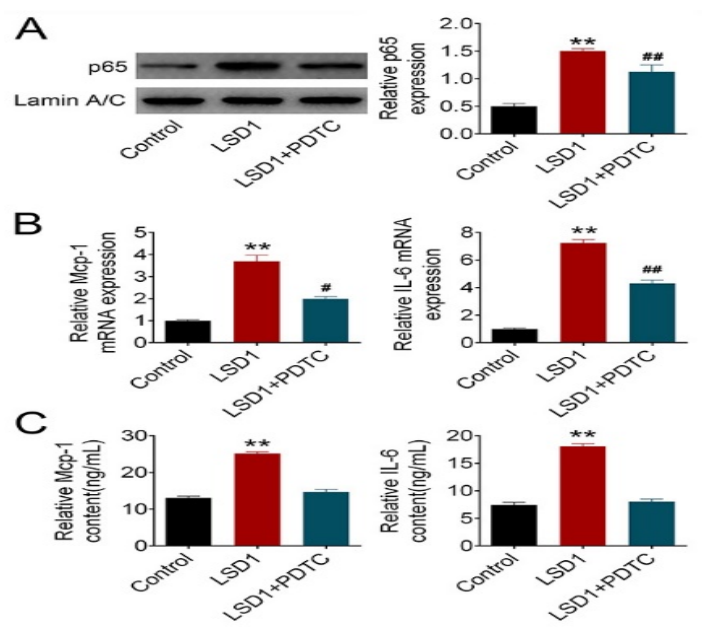

Figure 4: LSD1 exacerbated the inflammatory response in VSMCs by activation of NF-kB. (A) The protein level of p65 in VSMCs transfected with LSD1 overexpression plasmid and treated with PDTC was determined by Western blot. Levels of MCP-1 and IL-6 in VSMCs transfected with LSD1 overexpression plasmid and treated with PDTC were determined by qRT-PCR (B) and ELISA (C); ${ }^{* *} p<0.01$, compared to control group; $\# p<0.05$, compared to LSD1 group; $\#$ \# $<0.01$, compared to LSD1 group

\section{DISCUSSION}

Excessive inflammatory response of VSMCs is a contributing factor to various vascular diseases $[3,10]$. Therefore, it is critical to elucidate the regulatory mechanism of excessive inflammation in VSMCs, which may contribute to investigate novel targets for cardiovascular diseases therapy. In some studies, LSD1 was shown to be involved in the regulation of inflammatory response, suggesting that LSD1 might regulate the inflammatory response of VSMCs.

Increasing studies have reported that activation of p-LSD1 contributes considerably to many diseases. For example, Boulding et al [11] found that the activation of $p$-LSD1was critical for epithelial-to-mesenchymal transition activity and chemotherapy resistance of breast cancer cells. In addition, p-LSD1 expression level induced by $\mathrm{PKCa}$ is involved in circadian rhythmicity [12]. This study firstly showed that the p-LSD1 level was increased in VSMCs that treated with LPS. Consistently, Kim et al [9] found that p-LSD1 was activated by $\mathrm{PKC \alpha}$ in the inflammatory response. Therefore, p-LSD1 was activated during the inflammatory process of VSMCs.

To further study the role of LSD1 in the inflammatory response of VSMCs, the effects of LSD1 on the levels of inflammatory factors MCP. 1 and IL-6 and activation of NF-KB were determined. Knockdown of LSD1 decreased the levels of MCP-1, IL-6, and p65. These results are different from studies from Janzer et al [13] and Liu et al [14]. Janzer et al. found that downregulation of LSD1 increased inflammatory response in cancer cells via demethylation of lysine histone $\mathrm{H} 3$ [13]. Liu et al. also found that loss of LSD1 activity induced the production of pro-inflammatory cytokines and that this phenomenon may be related to demethylation of lysine histone H3 [14]. These differences may be caused by different regulatory mechanisms. The NF-KB signaling pathway is considered to be a prototypical proinflammatory pathway [15]. The results in our study showed that p65 levels were decreased by LSD1 knockdown, and the ratio of p65-me/p65 was increased after SET7/9 treatment, and these effects were abolished by overexpression of LSD1. These findings suggested that knockdown of LSD1 caused the inhibition of NF-kB activation via demethylation of p65. These results are consistent with results of Kim et al [9], revealing thatp-LSD1 could cause p65 demethylation.

In addition, lysine-specific demethylase 2A (KDM2A) also repressed NF-KB activity through p65 demethylation at K218 and K221 methylation sites[16]. LSD1, also known as KDM1A, along with KDM2A, are from the same family of molecules. It seems that LSD1 may also regulate p65 demethylation at K218 and K221 methylation sites, and thereby regulate NF-KB activation.

Finally, this study studied whether LSD1 could affect the inflammatory response by the regulation of NF-kB. The results showed that MCP-1 and IL-6 levels were increased by LSD1 
overexpression, and this effect was reversed by PDTC, an inhibitor of NF-kB activation. Accumulating evidences showed that NF-KB activation promoted inflammatory responses $[17,18]$. In general, LSD1 phosphorylation was activated during inflammatory response of VSMCs, and LSD1 promoted the activation of NF-KB via demethylation of p65, which then exacerbated the inflammatory response.

Excessive inflammation of VSMCs may be a critical component of cardiovascular disease. A mechanism by which LSD1 contributes to excessive inflammation of VSMCs was defined in this study. Thus, LSD1 may be a potential target for the treatment of cardiovascular diseases.

\section{CONCLUSION}

Lysine demethylase $1 \mathrm{~A}$ exacerbates LPSinduced inflammation of VSMCs through modulation of NF-kB activation via p65 demethylation. Thus, LSD1 may be a potential target for the treatment of cardiovascular diseases.

\section{DECLARATIONS}

\section{Conflict of interest}

No conflict of interest is associated with this work.

\section{Contribution of authors}

We declare that this work was done by the authors named in this article and all liabilities pertaining to claims relating to the content of this article will be borne by the authors. ZQQ and WHL conceived and designed the experiments, $\mathrm{LL}$ analyzed and interpreted the results of the experiments, MLS performed the experiments.

\section{Open Access}

This is an Open Access article that uses a funding model which does not charge readers or their institutions for access and distributed under the terms of the Creative Commons Attribution License (http://creativecommons.org/licenses/by/ 4.0) and the Budapest Open Access Initiative (http://www.budapestopenaccessinitiative.org/rea d), which permit unrestricted use, distribution, and reproduction in any medium, provided the original work is properly credited.

\section{REFERENCES}

1. Mantella LE, Quan A, Verma S. Variability in vascular smooth muscle cell stretch-induced responses in $2 D$ culture. Vasc Cell 2015; 7(1): 7.

2. Owens GK, Kumar MS, Wamhoff BR. Molecular regulation of vascular smooth muscle cell differentiation in development and disease. Physiol Rev 2004; 84(3): 767-801.

3. Qiu J, Zheng Y, Hu J, Liao D, Gregersen H, Deng X, Fan $Y$, Wang G. Biomechanical regulation of vascular smooth muscle cell functions: from in vitro to in vivo understanding. J $R$ Soc Interface 2014; 11(90): 20130852.

4. Chen J, Hu Q, Zhang B, Liu X, Yang S, Jiang H. Role of miR-134 in angiotensin II-induced vascular cell pathological changes in atherosclerosis. Tropical Journal of Pharmaceutical Research 2019; 18(5): 967973.

5. Ji Y, Liu J, Wang Z, Li Z. PPARgamma agonist rosiglitazone ameliorates LPS-induced inflammation in vascular smooth muscle cells via the TLR4/TRIF/IRF3/IP-10 signaling pathway. Cytokine 2011; 55(3): 409-419.

6. Zhong $Y$, Liu T, Guo Z. Curcumin inhibits ox- $L D L$-induced $M C P-1$ expression by suppressing the p38MAPK and NF-kappaB pathways in rat vascular smooth muscle cells. Inflamm Res 2012; 61(1): 61-67.

7. Shi Y, Lan F, Matson C, Mulligan P, Whetstine JR, Cole $P A$, Casero RA, Shi Y. Histone demethylation mediated by the nuclear amine oxidase homolog LSD1. Cell 2004; 119(7): 941-953.

8. Yang $Y T$, Wang $X$, Zhang $Y Y$, Yuan WJ. The histone demethylase $L S D 1$ promotes renal inflammation by mediating TLR4 signaling in hepatitis $B$ virus-associated glomerulonephritis. Cell Death Dis 2019; 10(4): 278.

9. Kim D, Nam HJ, Lee W, Yim HY, Ahn JY, Park SW, Shin HR, Yu R, Won KJ, Bae JS et al. PKCalpha-LSD1-NFkappaB-Signaling Cascade Is Crucial for Epigenetic Control of the Inflammatory Response. Mol Cell 2018; 69(3): 398-411.e396.

10. Robinson JG, Davidson MH. Can We Cure Atherosclerosis? Rev Cardiovasc Med 2018; 19(S1): s20-s24.

11. Boulding T, McCuaig RD, Tan A, Hardy K, Wu F, Dunn J, Kalimutho M, Sutton CR, Forwood JK, Bert AG et al. LSD1 activation promotes inducible EMT programs and modulates the tumour microenvironment in breast cancer. Sci Rep 2018; 8(1): 73.

12. Nam HJ, Boo K, Kim D, Han DH, Choe HK, Kim CR, Sun W, Kim H, Kim K, Lee H et al. Phosphorylation of LSD1 by PKCalpha is crucial for circadian rhythmicity and phase resetting. Mol Cell 2014; 53(5): 791-805.

13. Janzer A, Lim S, Fronhoffs F, Niazy N, Buettner R, Kirfel J. Lysine-specific demethylase 1 (LSD1) and histone deacetylase 1 (HDAC1) synergistically repress proinflammatory cytokines and classical complement 
pathway components. Biochem Biophys Res Commun 2012; 421(4): 665-670.

14. Liu D, Zempleni J. Low activity of LSD1 elicits a proinflammatory gene expression profile in riboflavindeficient human T Lymphoma Jurkat cells. Genes Nutr 2014; 9(5): 422.

15. Lawrence T. The nuclear factor NF-kappaB pathway in inflammation. Cold Spring Harb Perspect Biol 2009; 1(6): a001651.

16. Lu T, Jackson MW, Wang B, Yang M, Chance MR, Miyagi M, Gudkov AV, Stark GR. Regulation of NFkappaB by NSD1/FBXL11-dependent reversible lysine methylation of p65. Proc Natl Acad Sci U S A 2010; 107(1): 46-51.

17. Lawrence $T$, Bebien $M$, Liu GY, Nizet $V$, Karin $M$. IKKalpha limits macrophage NF-kappaB activation and contributes to the resolution of inflammation. Nature 2005; 434(7037): 1138-1143.

18. Xia YF, Ye BQ, Li YD, Wang JG, He XJ, Lin X, Yao X, Ma $D$, Slungaard A, Hebbel RP et al. Andrographolide attenuates inflammation by inhibition of NF-kappa $B$ activation through covalent modification of reduced cysteine 62 of p50. J Immunol 2004; 173(6): 4207-4217. 